\title{
Energy Saving Model and Simulation Test Based on 6LoWPAN Wireless Sensor Network
}

\author{
Yunyi Zhang ${ }^{1, *}$, Yong $\mathrm{Li}^{1}$, Ran Zhang ${ }^{1}$ and Wei Wei ${ }^{2}$ \\ ${ }^{I}$ College of Computer and Communication Engineering, Zhengzhou University of Light Industry, Zhengzhou 450002, \\ China \\ ${ }^{2}$ School of Computer Science and Engineering, Shaanxi Key Laboratory for Network Computing and Security Technolo- \\ gy, Xi'an University of Technology, Xi'an 710048, China
}

\begin{abstract}
This paper designs the mathematical model of fiber line power, optimization of wireless sensor network node and multi distributed IP, and uses C language to program the model, finally get a new energy-saving mechanism model of wireless sensor network. Combined with the distributed IPv6 technology and IEEE802.15.4 protocol, this paper designs a new 6LoWPAN wireless sensor network energy-saving mechanism, which realizes the seamless splicing energy-saving effect between wireless sensor network and the internet, and based on this develops Contiki integrated environment of wireless sensor network energy-saving test. Finally this paper uses Firefox browser with B/S architecture to test energysaving mechanism. Through the test we found that between two nodes of wireless sensor network, they can effectively realize sending and receiving of UDP data, and through IPv6 gateway it can connect with internet, and complete collection and transmission of data. Through the simulation calculation of power consumption, in accepting, transmission and idle periods, the wireless sensor network can save energy effectively, which reduces the splicing cost of internet and wireless sensor network. It provides the technical reference for the design of wireless sensor network.
\end{abstract}

Keywords: 6LoWPAN network, Distributed IPv6, IEEE802.15.4, C language, Contiki integration.

\section{INTRODUCTION}

Wireless sensor networks are mostly local area network with ad hoc network form. It can only access and process the monitored information in the dedicated control center. IP network is widely used, if such local area wireless sensor network can be accessed internet backbone network, it will bring more convenience to users. According to the complex gateway of internet protocol development, there are three main problems for wireless access to the internet: firstly it needs a dedicated adapter; secondly the data package of the existing internet format occupies large space with big power, and it can't meet the requirements of wireless sensor network terminal; thirdly when connecting many embedded devices with IP network, due to the limited IP address for current IPv4 protocol, it often faces addresses shortage [1]. Due to function advantage, 6LoWPAN can solve these problems very well, and can reduce the energy consumption of the network. Based on this, this paper designs the energysaving mechanism of wireless sensor network, and tests the mechanism, finally realizes the energy-saving optimization design of wireless sensor network.

\section{OVERVIEW OF 6LOWPAN WIRELESS SENSOR NETWORK ENERGY-SAVING PRINCIPLE}

With the development of computer network technology, more and more devices need access to network. The 6LoWPAN working group of the Internet Engineering Task Force will be dedicated to apply IPv6 in IEEE 802.15.4, so as to realize the seamless connection between wireless sensor network and IP network [2]. The energy-saving mechanism of 6LoWPAN wireless sensor network is related to network configuration and energy-saving of sensor terminal [12]. This paper combines the two aspects to establish a new energy-saving mechanism. The basic principle is as follow:

\subsection{Main Energy-saving Mode of 6LoWPAN Wireless Sensor Network}

6LoWPAN wireless sensor network is a joint product of wireless sensor network technology, IPv6 technology and network technology. The energy-saving way is realized mainly through the network configuration and energy-saving of wireless sensor terminal [3-10].

(1) Network configuration: 6LoWPAN technology solves the internet accessing problem of perception layer and wireless sensor node, and realizes the IP implementation of wireless sensor terminal. Fig. (1) shows the built wireless sensor network using 6LoWPAN technology. 


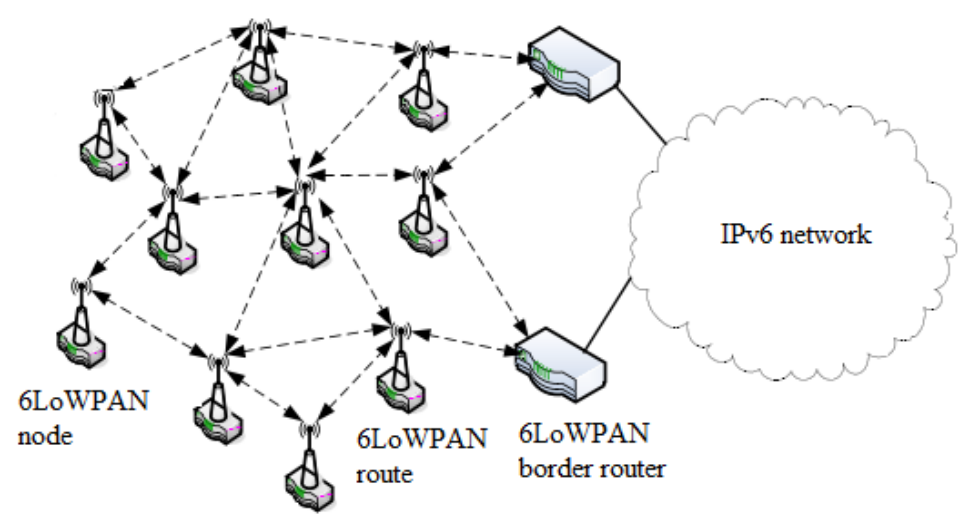

Fig. (1). Energy-saving mechanism of distributed network.

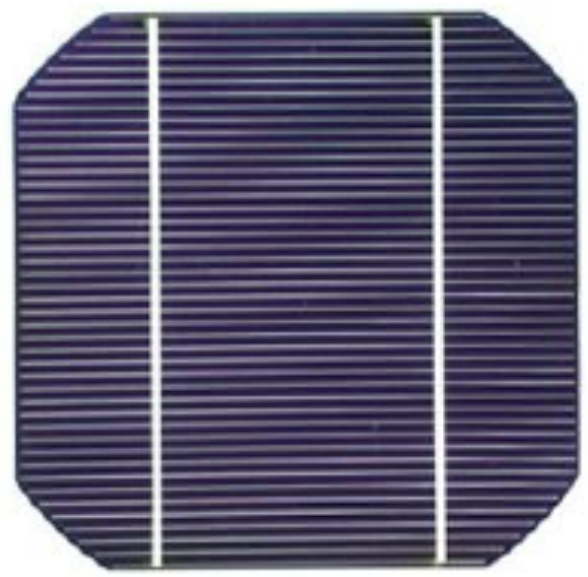

Fig. (2). Mono-crystal line solar cell.

Fig. (1) shows the energy-saving mechanism of 6LoWPAN wireless sensor network node based on distributed network nod [11]. The distributed network uses Ipv6 distributed network system, and uses the boundary router to connect the sensor network with Ipv6 network system. Between nodes it uses 6LoWPAN router to realize node information exchange and energy saving.

(2) Sensor terminal: sensor terminal energy consumption is also one of the 6LoWPAN wireless sensor network energy consumption. This paper mainly uses two kinds of sensor terminal solar cell to realize the design of low power consumption, including mono-crystal line and polycrystalline solar cell.

\section{(a) Mono-Crystal Line Solar Cell}

Mono-Crystal Line solar cell uses Mono-Crystal Line silicon to produce battery; its basic structure is as shown in Fig. (2).

Mono-Crystal Line cell has stable power performance, and it is one of the most used solar cells. Compared with polycrystalline silicon battery, it has higher efficiency of solar energy conversion, but it also has high requirements for material, so the production cost is also higher.

\section{(b) Polycrystalline silicon solar cells}

Compared with Mono-Crystal Line silicon solar cells, the advantage of polycrystalline silicon solar cells is saving material, and the disadvantage is lower transformation efficiency.

As shown in Fig. (3), polycrystalline silicon solar cell uses chemical gas phase deposition, and it is easy to manufacture with low cost. Its performance is higher than that of Mono-Crystal Line silicon solar cells, so it has been widely applied in the solar energy-saving battery.

\subsection{Overview of 6LoWPAN Wireless Sensor Network Energy-saving Mechanism}

Wireless sensor network can be divided into different types according to the different applications, but the wireless sensor network nodes is basically the same, which has four main modules, including power supply module, wireless 


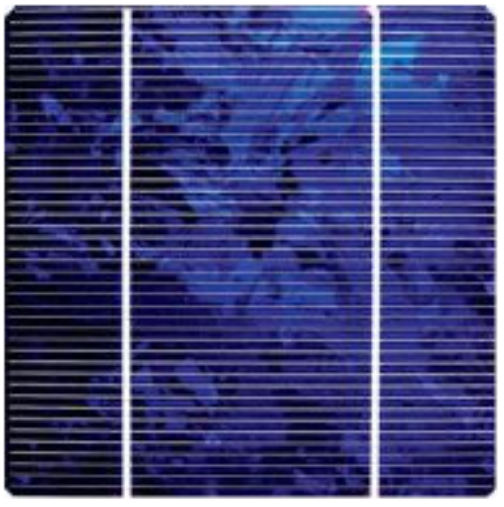

Fig. (3). Polycrystalline silicon solar cell.

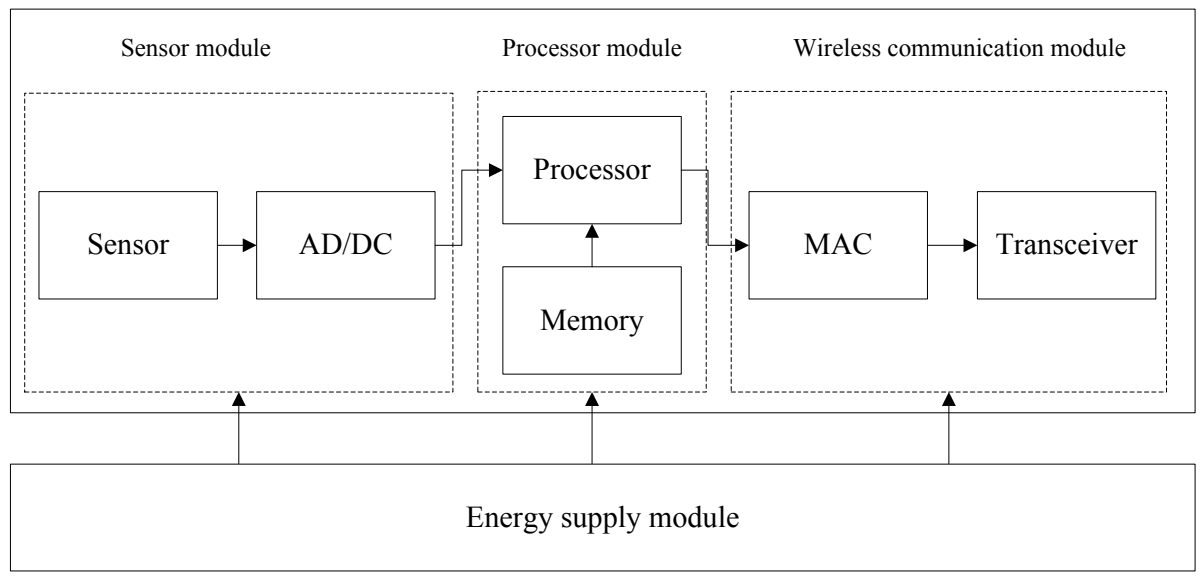

Fig. (4). Architecture of the sensor node.

communication module, processor module, sensor module. The concrete frame is as shown in Fig. (4).

Among them, the energy supply module is the essential part of all electronic systems, it is the power center of the whole network and responsible for providing the necessary energy, and the main energy consumption is the three big modules. When using 6LoWPAN technology it can achieve energy saving from the following two aspects:

\subsection{Multi Task Kernel Driven by Event}

6LoWPAN uses the Contiki integrated development environment. This driver model can make multiple tasks share one stack, without occupying independent stack for each task. Each task occupies only a few bytes of RAM, greatly saving storage space, more suitable for wireless sensor network.

\subsection{Low Power Wireless Sensor Network Protocol Stack}

Contiki provides a complete IP network and the low power wireless network protocol stack [13]. For the IP protocol stack, it supports IPv4 and IPv6, including some simplified web tools, such as Telnet, HTTP and web services. Integrated environment reduces network energy consumption.

\section{MATHEMATICS MODEL AND ALGORITHM OF 6LOWPAN WIRELESS SENSOR NETWORK ENER- GY-SAVING MECHANISM}

The establishment of wireless sensor energy-saving mechanism can greatly reduce energy consumption, save computer hardware/software and network communication resources, which has an important significance for the design of wireless sensor network.

\subsection{Energy Consumption of 6LoWPAN Wireless Sensor Network}

In order to improve the transmission efficiency of 6LoWPAN, this paper uses optical fiber transmission in wireless network communication line [14]. Power consumption of network signal transmission in the optical fiber line is mainly related to optical system loss and dispersion. The maximum effective transmission distance of optical loss is as shown in formula 1 .

$$
g=\frac{G_{m}-G_{n}-G_{c}-2 K_{p}-L_{p}}{\lambda_{a}-\lambda_{b}}
$$


Among them, $G_{m}$ is the luminous power for completely loss, $G_{n}$ is receiving sensitivity without security loss, $Y_{n}$ is the luminous flux rate, $\lambda_{a}, \lambda_{b}$ respectively is the light loss coefficient, $K_{p}$ is loss at the interface, $L_{p}$ is the maximum communication rate of cable, in which the dispersion limited expression is as shown in formula 2 .

$L=\frac{S}{k}$

$L$ is the dispersion limited distance, $S$ is the largest dispersion, $k$ is dispersion coefficient. In the actual construction process, setting up of fiber optic lines needs to consider the maximum effective transmission distance and dispersion limit, so the maximum effective range is as follows:

$\widehat{\mathrm{L}}=\max (g, L)$

Formula 3 can effectively calculate the energy consumption of 6LoWPAN wireless sensor network in the actual building process, which provides basis for the establishment of energy-saving mechanism.

\subsection{LOWPAN Wireless Sensor Network Nodes Optimi- zation}

In the design of 6LoWPAN wireless sensor network, network nodes processing can improve network performance and reduce the energy consumption of the network [15]. This paper established a mathematical model of independent nodes by using the least square method. Assuming that independent sequence of 6LoWPAN network node is $\left(x_{1}, y_{1}\right),\left(x_{2}, y_{3}\right) \ldots . .\left(x_{n}, y_{n}\right)$, so we can get:

$y_{i}=\lambda+n x_{i}+X_{\sigma i}$

In the formula 4, using the least square method to optimize independent node sequence, the least squares estimation is as shown in formula 5.

$$
\begin{aligned}
& \hat{n}=\frac{\sum_{i=1}^{m}\left(x_{i}-\bar{x}\right)\left(y_{i}-\bar{y}\right)}{\sum_{i=1}^{m}\left(x_{i}-\bar{x}\right)^{2}} \\
& -\bar{x}=\frac{1}{m} \sum_{i=1}^{m} x_{i}, \bar{y}=\frac{1}{m} \sum_{i=1}^{m} y_{i}
\end{aligned}
$$

Maximum likelihood estimation values of 6LoWPAN network independent nodes energy consumption in $X_{\sigma}$ can be expressed as:

$$
\psi=\sqrt{\frac{1}{m} \sum_{i=1}^{m} x_{\sigma_{i}}^{2}}
$$

Optimization distance of 6LoWPAN independent network node can be expressed as:

$f\left(S_{d}\right)=\frac{1}{\psi \sqrt{2 \pi}} e^{\frac{-\left[S_{d}-\left(P_{f}+Z_{f}+Z_{j}-S_{l}-S_{d}\right)\right]}{2 \psi^{2}}}$

$S_{d}$ is optimization distance, covariance and distance function. When $S_{d} \geq S d_{0}$, the performance and the energysaving state of 6LoWPAN wireless networks achieve optimal, so we can carry on the design of network transmission line.

\subsection{Mathematical IP Multiple Distributing Model of 6LoWPAN Wireless Sensor Network}

Multi distributed network can realize the transmission of high capacity information; its core technology is the application of IP multi distribution technology [16]. In order to optimize multiple distributed networks it needs to design space distribution of IP network node. Assuming that the distance of multiple distributions IP network node is $y$, its expression is as follows:

$\int_{t_{1}}^{t_{2}} \sqrt{\left(\frac{d u}{d t}\right)^{2}+\left(\frac{d v}{d t}\right)^{2}} d t=y$

So it can use pan function to calculate extreme value of distribution node.

$y(u, v)=\frac{1}{2} \int_{y_{1}}^{y_{2}}\left(u \frac{d u}{d t}-v \frac{d v}{d t}\right) d t$

By using the function variation principle, if

$$
J(u)=H u^{2}-2 f u \quad(H>0)
$$

$H, f$ are real constants, it can use multiple function variation principle to solve the minimum value point.

$J(\vec{u})=J\left(u_{1}, u_{2}, \cdots, u_{n}\right)=\frac{1}{2} \sum_{i=1}^{n} \sum_{j=1}^{n} a_{i j} u_{i} u_{j}-\sum_{i=1}^{n} b_{i} u_{i}$

In multi distribution IP network system, network node supports super nodes. This function can search better performance of node in the area, then use DHT algorithm to form an overlay network, forming subnet system of super nodes [17]. The super node can also choose the standby node, when a super node is in failure, it can choose another node as a backup node, which saves node resources and energy consumption of the network, as shown in Fig. (5).

As shown in Fig. (5), it can greatly improve the utilization rate of network nodes by using distributed IPv6 network, form wide coverage range of wireless network, and effectively reduce the energy consumption, which can be applied in network energy-saving mechanism of 6LoWPAN. 


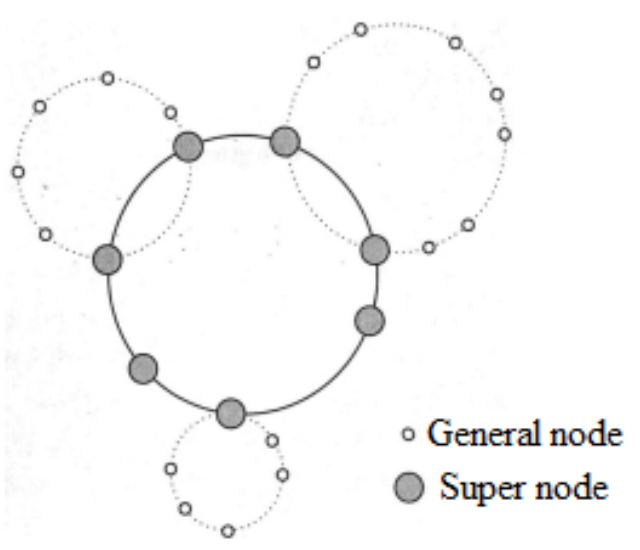

Fig. (5). Schematic diagram of the novel IPv6 wireless sensor network node.

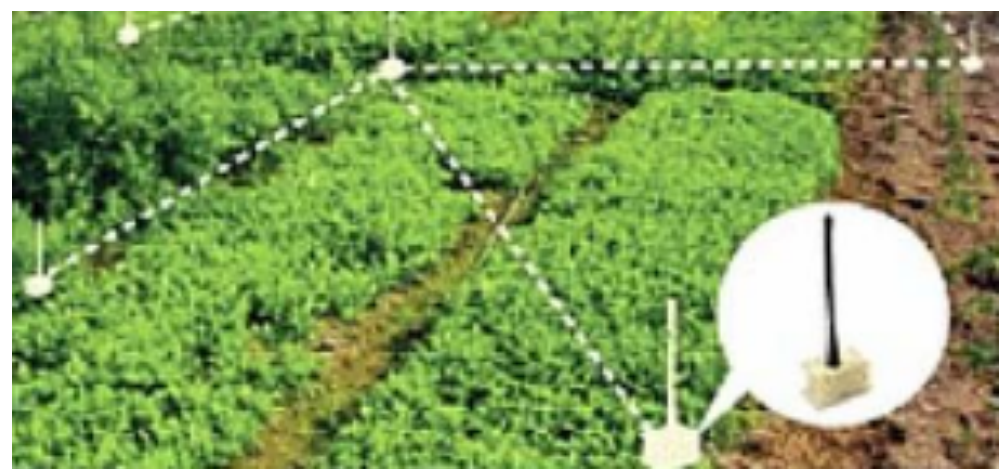

Fig. (6). Wireless sensor network layout.

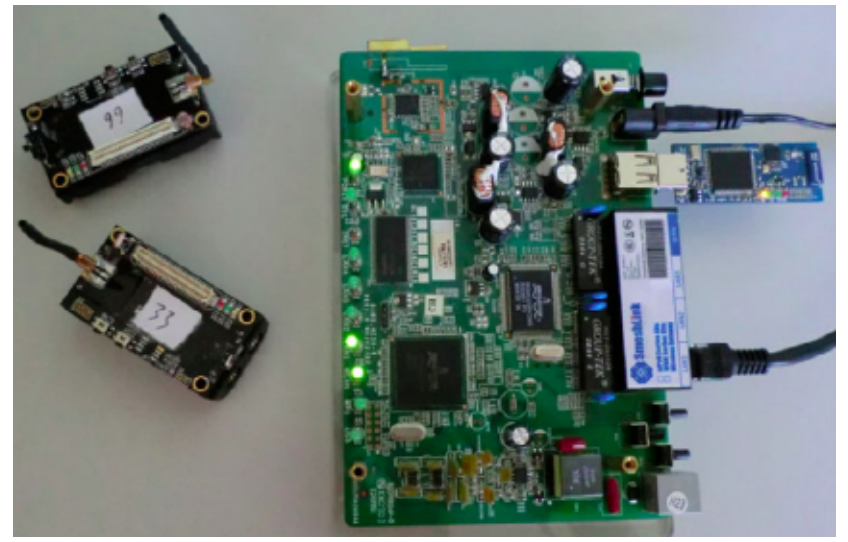

Fig. (7). The experiment hardware diagram.

\section{ENERGY-SAVING EXPERIMENT STUDY OF 6LoWPAN WIRELESS SENSOR NETWORK}

In order to test the effectiveness and reliability of energysaving mechanism mathematical model for 6LoWPAN wireless sensor network designed in section second, this paper combines the wireless sensor network with IPv6, introduces IEEE802.15.4 technology into the network design, and does seamless splicing experiment between wireless sensor networks and the internet.

In order to verify the energy-saving effect of 6LoWPAN wireless sensor network, this paper sets up a wireless sensor network, as shown in Fig. (6). The wireless sensor network terminal uses polycrystalline silicon solar cell $[18,19]$. The bottom layer uses IEEE802.15.4 and MAC layer, between the network layer and IEEE protocol it adds 6LoWPAN adaptation layer, transport layer uses the UDP protocol and CoAP protocol. The HTTP gateway can use CoAP protocol to read, delete and modify the source data.

\subsection{The Development Contiki Integrated Environment}

The experiment uses suite developed by Mei Xin Lin Ke Company, which are developed on Contiki platform. The kit supports IEEE802.15.4 physical transmission protocol and IPv6 development environment, the hardware device is as shown in Fig. (7). 


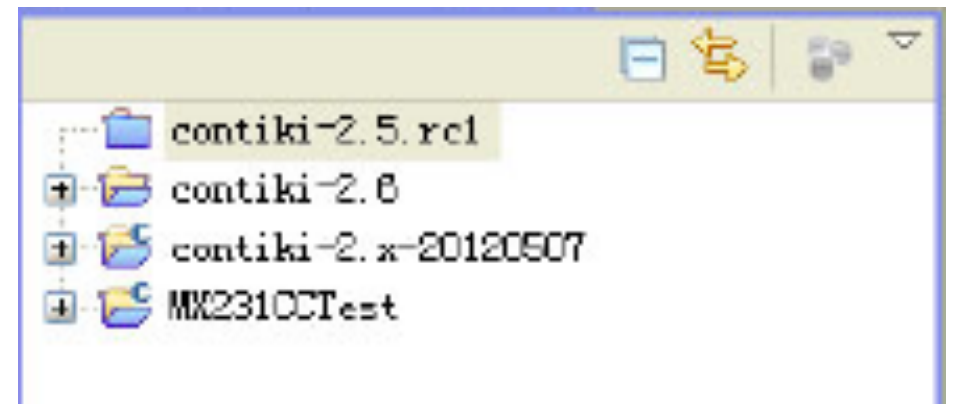

Fig. (8). The ContikiStudio integrated environment development.

Fig. (7) shows the experimental hardware diagram of 6LoWPAN wireless sensor network designed in this paper. The hardware part is mainly composed of transmitting node, transmission module, and transmission gateway [19]. The software part is mainly composed of Contikistudio integrated development platform. The installation of software integration environment is as shown in Fig. (8).

The Contiki integrated development environment is a multi task operating environment system, which has better portability and open source performance, and occupies less memory in the process of operation, so it is very suitable for application in wireless sensor networks and embedded operating system [20, 21]. Contiki uses C language program, with lower hardware requirements, and it suitable for use in a variety of micro computer, computer, and PC SCM. Based on 6LoWPAN wireless network, this paper develops the data transmission procedure, and the main data acquisition procedures of sensor end are as follows:

private void get temperature()

\{

Sql Connection con $=$ new Sql Connection(DBHelper.connString.ToString());

con.Open();

string sql = "select a.url,a.port,b.mid,b.uri from sh_mote as $\mathrm{a}$,sh_typemote as b where

a. mid $=$ b.mid and a.flag = '1' and b.flag=' 1 ' and b.tflag='3'";

SqlCommand $\mathrm{cmd}=$ new SqlCommand(sql, con);

Inserting the collected wireless sensor data into the internet database, the procedure is as follows:

private void insertdatatemperature(int mid, string str, DateTime nowtime)

\{

Sql Connection con $=$ new SqlConnection(DBHelper.connString.ToString());

con.Open(); string sql $=\quad$ string.Format("insert into sh_temperature(mid,temperature,nowtime)

values('\{0\}','\{1\}','\{2\}')", mid, str, nowtime);

SqlCommand $\mathrm{cmd}=$ new SqlCommand(sql, con);

SqlDataReader $\mathrm{sdr}=$ cmd.ExecuteReader();

sdr.Close();

con.Close();

\}

Database data needs to be sent to wireless gateway in CoAP data packets, the program is:

RESOURCE(light, METHOD_GET, "light");

Void light handler(REQUEST* request, RESPONSE* response)

\{

char buf[32];

int16_t light $=$ NULLDATA;

light $=$ sensor_light_get();

sprintf(buf, "\%d", light);

char etag[4] = "ABCD";

\subsection{Wireless Sensor Network Test for 6LOWPAN Ener- gy-saving Mechanism}

In order to test the 6LoWPAN energy-saving mechanism designed in this paper, this paper uses the Firefox browser to test the system. The Firefox browser uses the B/S frame, and the frame structure is shown in Fig. (9).

As shown in Fig. (9) it represents the schematic architecture of Firefox browser testing system designed in this paper. This system is composed of browser, web server, IPv6 intelligent gateway, MX231CC nodes. The accessing of browser uses HTTP protocol, and nodes communicate through IPv6 gateway and CoAP protocol [22]. So it realizes the user can use the internet to access the sensor data resources. 


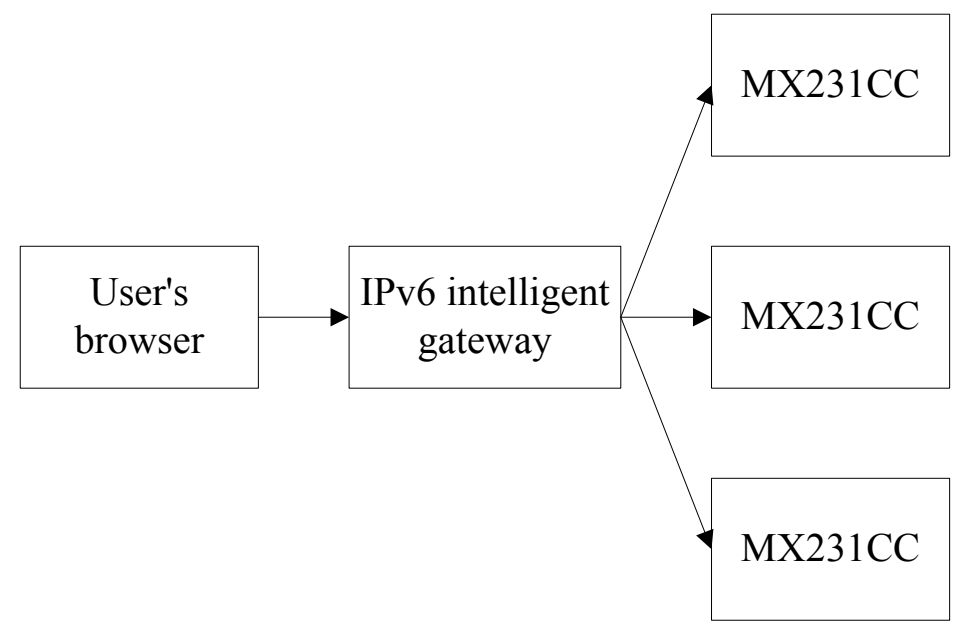

Fig. (9). Structure diagram of 6LoWPAN with B/S architecture.

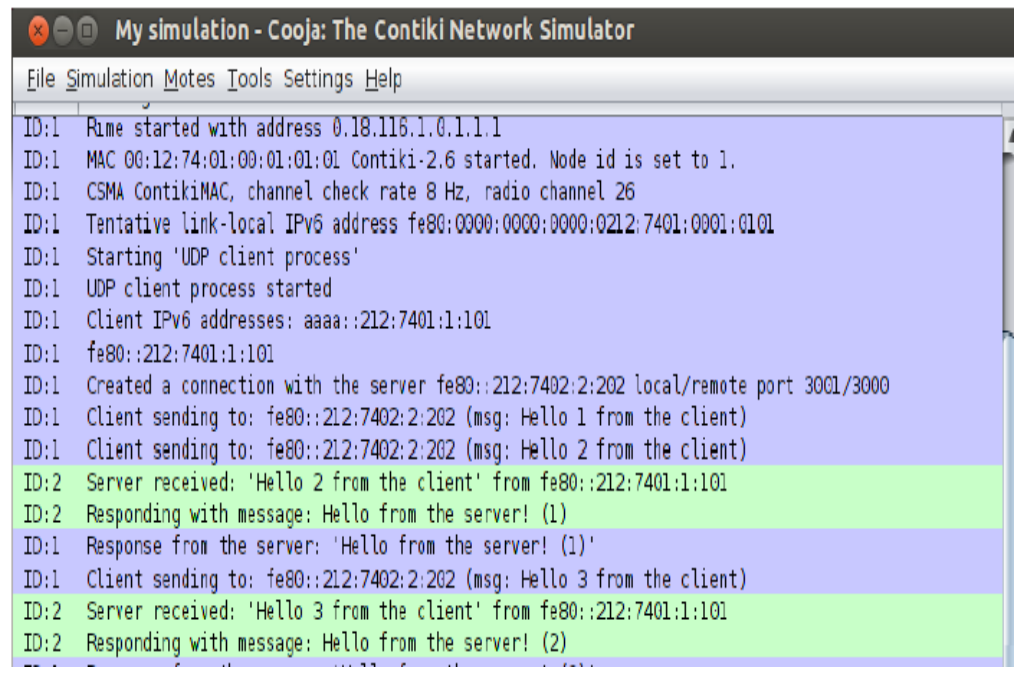

Fig. (10). The communication design between the two 6LoWPAN.

In order to verify the normal of network communication, it uses test software to simulate the communication between two 6LoWPAN wireless sensor network nodes, and uses IPv6 to send and receive UDP data packets. As shown in Fig. (10), for two nodes, one is as server, and the other is as a Client. After the establishment of digital connection, node 1 uses UDF to send data to node 2 [23]. It means that two 6LoWPAN sensor nodes can go UDP data transmission as IPv6 host.

This test simulates a complete course of 6LoWPAN wireless sensor network established by two 6LoWPAN wireless sensor nodes through neighbor discovery protocol [24]. This network uses RPL routing as shown in Fig. (11). By tests we found, the wireless sensor network node 2 can realize data acquisition, and send to node 1 through the 6LoWPAN wireless network. Node 1 saves data in the form of UDP, which verified the stability and reliability of network communication.
The node of wireless sensor network is composed of four parts, except the power supply unit, the other is energy consuming unit, and the wireless communication unit is the largest energy consumption unit [25]. This paper uses 6LoWPAN to test and obtain energy consumption of each function unit. The energy-saving data is as shown in Fig. (12).

In general, the node wireless communication unit has four states: send, receive, idle, and sleep. As shown in Fig. (12), in order to show the energy-saving of nodes directly, the energy-saving data is listed in Table $\mathbf{1}$.

From Table 1 we can see, when receiving and sending data, the energy consumption of the node is the maximum. When the device is in idle, monitoring channel is still in working condition, so the energy consumption is relatively large, and is relatively low at other time. From the energysaving effect we can see, in the links of more energy 


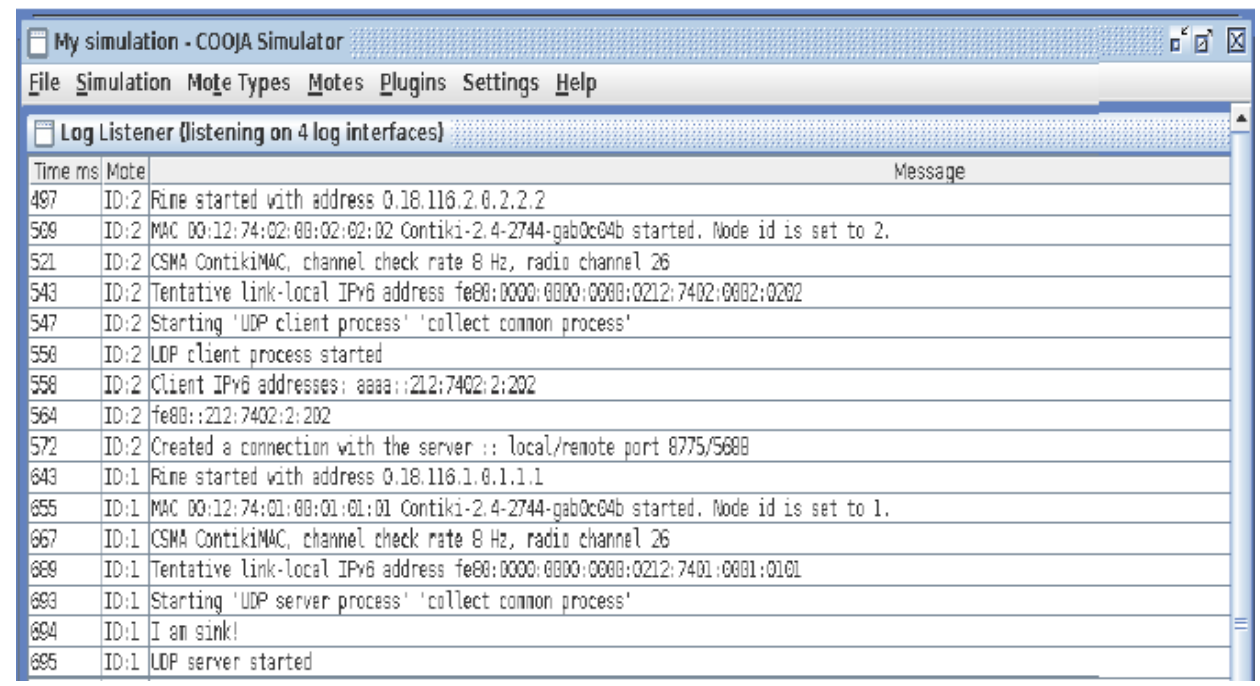

Fig. (11). Wireless sensor network routing test protocol.

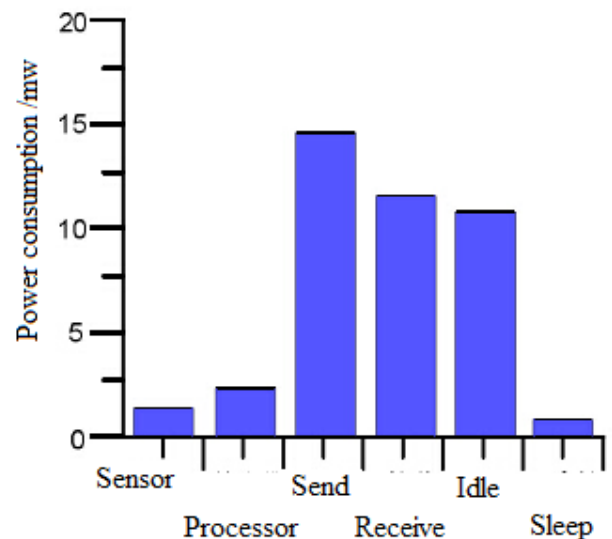

Fig. (12). Each unit energy-saving for the node.

Table 1. The statistics of 6LoWPAN wireless sensor network energy-saving.

\begin{tabular}{|c|c|c|c|}
\hline Test Items & $\begin{array}{c}\text { Common Wireless Sensor Network } \\
\text { Energy Consumption (mV) }\end{array}$ & $\begin{array}{c}\text { 6LoWPAN Sensor } \\
\text { Network Energy Consumption (mV) }\end{array}$ & Network Energy-Saving (mV) \\
\hline \hline Sensor & 4.64 & 3.12 & 1.52 \\
\hline Processor & 5.04 & 2.11 & 14.93 \\
\hline Send & 26.73 & 12.52 & 12.86 \\
\hline Receive & 23.07 & 10.21 & 12.10 \\
\hline Idle & 21.62 & 9.52 & 1.21 \\
\hline Sleep & 2.53 & 1.32 & \\
\hline
\end{tabular}

consuming, energy-saving effect of 6LoWPAN is more obvious, and maximum energy-saving can reach $14.21 \mathrm{mV}$. It indicates that the 6LoWPAN can maximize use internet resources, reduce the energy consumption, and improve the efficiency of network communication and transmission.
Fig. (13) shows the test on the three nodes in 6LoWPAN wireless sensor network, and gets the average delay simulation curve [26]. From the chart we can see, along with the increase of multi distribution IP area, the average network delay reduces gradually, thus verifies the validity and relia- 


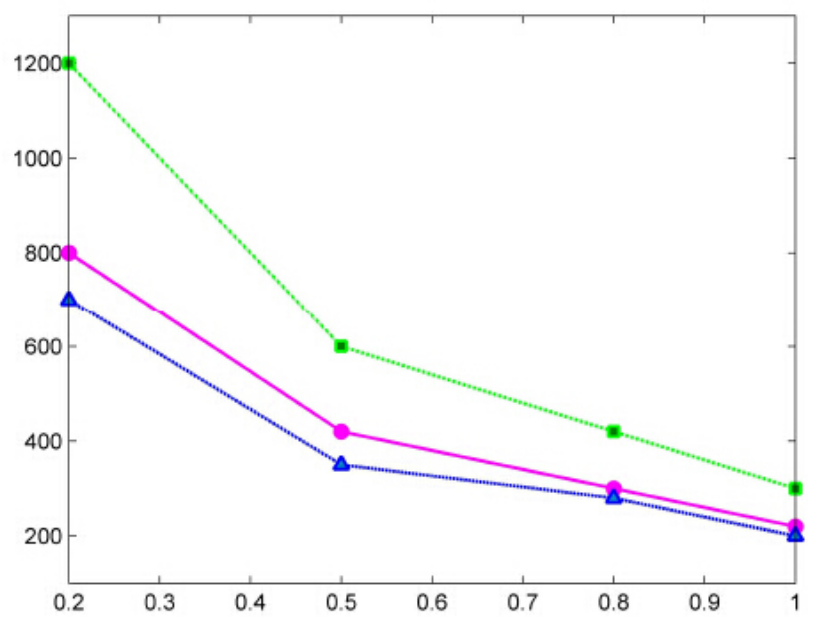

Fig. (13). The average delay of nodes.

bility of multi distributed IP 6LoWPAN energy-saving mechanism.

\section{SUMMARY}

This paper combines the wireless sensor network with distributed multi IPv6, and introduces IEEE802.15.4 technology into the network design, and seamless misaiming wireless sensor networks with the internet, finally obtains a new 6LoWPAN wireless sensor network energy saving mechanism. The mechanism uses combined power of optical fiber line, wireless sensor network node distribution optimization and multi distributed IP technology to design energysaving model, and uses $\mathrm{C}$ language to program the algorithm. In order to test the effectiveness and reliability of the energy-saving mechanism, this paper builds energy-saving wireless sensor network, and develops the integrated Contiki environment, finally uses Firefox browser with B/S architecture to test the energy-saving mechanism. Through the test we found that when sending, receiving, idle and sleep for wireless sensor network, 6LoWPAN wireless sensor network can realize the network energy-saving.

\section{CONFLICT OF INTEREST}

The authors confirm that this article content has no conflict of interest.

\section{ACKNOWLEDGEMENTS}

We would like to thank the anonymous reviewers for their valuable comments. We also sincerely appreciate Prof. Yong Qi for his valuable advices and helping. This program is supported by Scientific Research Program Funded by key scientific and technological project of Henan Province (No. 142102210081) and Shaanxi Provincial Education Department (Program No.2013JK1139) and Supported by China Postdoctoral Science Foundation (No.2013M542370) and supported by the Specialized Research Fund for the Doctoral Program of Higher Education of China (Grant No. 20136118120010). And this project is also supported by
NSFC Grant (Program No. 11301414 and No.61272283 and No.61272283 and No.61472318) .

\section{REFERENCES}

[1] J. Ma, "Application and standards present situation of IPv6 technology in the ubiquitous network aware extension layer," Telecom Network Technology, vol. 2, no. 3, pp. 35-39, 2012.

[2] L. Jiang, and L. Hou, IPv6 and network, Beijing Industry Press, 2012, pp. 56-42.

[3] W. Wei, and Y. Qi, "Information potential fields navigation in wireless Ad-Hoc sensor networks," Sensors, vol. 11, pp. 47944807, 2011.

[4] W. Wei, P. Shen, Y. Zhang, and L. Zhang, "Information fields navigation with piece-wise polynomial approximation for highperformance OFDM in WSNs", Mathematical Problems in Engineering, vol. 2013, Article: 901509, 2013, DOI: 10.1155/2013/901509, Year: SCI:WOS:000316891600001.

[5] W. Wei, Q. Xu, L. Wang, X.H. Hei, P. Shen, W. Shi, and L. Shan, "GI/Geom/1 queue based on communication model for Mesh Networks", International Journal of Communication Systems, vol. 27, no. 11, pp. 3013-3029, 2014.

[6] W. Wei, Y. Qiang, and J. Zhang, "A bijection between latticevalued filters and lattice-valued congruences in residuated lattices", Mathematical Problems In Engineering, vol. 2013, Article ID: 908623, DOI: 10.1155/2013/908623, Year: 2013, SCI:WOS:000322476900001, EI: 20133416634635.

[7] W. Wei1, L. Zhang, D. Guo, and P. Shen, "Applications of information navigation method in wireless sensor networks", Journal on Communications, vol. 33, no. Z2, pp. 146-152, 2012.

[8] W. Wei, X.L. Yang, B. Zhou, and P. Shen, "Holes detection in anisotropic sensornets: topological methods", International Journal of Distributed Sensor Networks, vol. 2012, Article ID 135054, 2012. Doi:10.1155/2012/135054, SCI WOS:0003106-24300001, EI: 20124915745593.

[9] W. Wei, X. Yang, B. Zhou, and J. Feng, "Combined energy minimization for image reconstruction from few views", Mathematical Problems in Engineering, vol. 2012, Article ID 154630, 15 Doi:10.1155/2012/154630, SCI WOS:000310902600001, EI: 20124915745650), 2012.

[10] W. Wei, H. M. Srivastava, Y. Zhang, L. Wang, P. Shen, and J. Zhang, "A local fractional integral inequality on fractal space analogous to anderson's inequality", Abstract and Applied Analysis, Ariticle ID: 797561, DOI: 10.1155/2014/797561, Year: 2014.

[11] H. Wang, "Research on network technology and its development," Journal of Bohai University (Natural Science Edition), vol. 9, no. 9, pp. 265-268, 2012.

[12] X. Wang, and H. Qian, "State address configuration scheme of 6LoWPAN wireless sensor network," Application Research of Computers, vol. 3, no. 10, pp. 35-36, 2011. 
[13] Y. Wang, Z. Qian, X. Wang, and D. Sun, "Network addressing strategy for 6LoWPAN," Journal of Electronics \& Information Technology, vol. 3, no. 4, pp. 763-769, 2013.

[14] X. Zhang, and E. Bai, "Sensor gateway design based on 6LoWPAN," Electronic Science and Technology, vol. 3, no. 12, pp. 11-14, 2012.

[15] S. Chen, "Research on the mobile internet security technology," Information Safety and Communication Security, vol. 3, no. 8, pp. 35-39, 2011.

[16] W. Zhang, and H. Jiang, "Building a sound resource management system," Communication Management and Technology, vol. 11, no. 7, pp. 451-453, 2012.

[17] Y. Wu, B. Li, C. Sun, and X. Zheng, "Research on peer to peer network content search technology based on the Chord," Microcomputer, vol. 27, no. 1, pp. 164-167, 2011.

[18] Z. Hua, and G. Wang, "Improved P2P model based on pastry," Communication Technology, vol. 4, no. 3, pp. 96-99, 2011.

[19] X. Fang, J. Zhang, and X. Zhang, "Communication mechanism and network governance for instant network era," Modern Communication, vol. 6, no. 5, pp. 56-59, 2011.
[20] L. Chen, S. Mei, and Y. Chen, "Intelligent grid information security and its impact on the survival of power systems," Control Theory and Applications, vol. 29, no. 2, pp. 240-244, 2012.

[21] Y. Lu, D. Liu, and J. Liu, "Intelligent grid network information integration requirements and model analysis," Automation of Electric Power Systems, vol. 34, no. 8, pp. 1-4, 2012.

[22] J. Ni, G. He, and S. Chen, "Overview of USA smart grid assessment," Automation of Electric Power Systems, vol. 34, no. 8, pp. 9$13,2012$.

[23] J. Hu, "Jump strategy of strong smart grid," National Grid, vol. 2, no. 1, pp. 25-28, 2011

[24] X. Huang, P. Liu, S. Miao, and X. Wang, "Dynamic application of wireless sensor networks in power control," Power System Automation, vol. 31, no. 7, pp. 99-103, 2013.

[25] Q. Zhang, Y. Sun, T. Yang, and Z. Cui, "Application of wireless sensor network in intelligent power grid," Chinese Electric Power, vol. 43 , no. 6 , pp. 31-36, 2011

[26] X. Fan, X. Zheyuan, Z. Chen, S. Liu, and Z. Qu, "Research on video coding in wireless multimedia sensor networks," Journal of Communication, vol. 32, no. 9, pp. 137-146, 2011

(c) Zhang et al.; Licensee Bentham Open.

This is an open access article licensed under the terms of the Creative Commons Attribution Non-Commercial License (http://creativecommons.org/licenses/by-nc/3.0/) which permits unrestricted, non-commercial use, distribution and reproduction in any medium, provided the work is properly cited. 